\title{
Review: enteral nutrition reduces infections, need for surgical intervention, and length of hospital stay more than parenteral nutrition in acute pancreatitis
}

Marik PE, Zaloga GP. Meta-analysis of parenteral nutrition versus enteral nutrition in patients with acute pancreatitis. BMJ 2004;328:1407.

\section{$Q$ What is the efficacy and safety of enteral nutrition (EN) compared with total parenteral nutrition (PN) for patients with acute pancreatitis (AP)?}

\section{METHODS}

Data sources: Medline (1966 to January 2004), EMBASE/
Excerpta Medica, Cochrane Controlled Trials Register, and
Cochrane Database of Systematic Reviews; bibliographies of
relevant studies; and experts in the field.
Study selection and assessment: randomised controlled trials
(RCTs) that compared EN with PN in patients admitted to hospital
with AP, and included $\geqslant 1$ of the outcomes listed below.
Methodological quality of individual studies was assessed using
the 5 point Jadad composite scale.
Outcomes: infections (pneumonia, abdominal abscess,
pancreatic abscess, wound infection, or bloodstream infection),
non-infectious complications (adult respiratory distress syndrome,
multiorgan failure, acute pseudocysts, and pancreatic fistula),
need for surgical intervention, length of hospital stay, and
hospital mortality.

\section{MAIN RESULTS}

6 trials $(\mathrm{n}=263)$ met the selection criteria. 4 studies had poor quality (Jadad score $\leqslant 2$ out of 5 ). Meta-analysis was completed using a random effects model and intention to treat data from individual studies. Patients who received EN had a lower risk of infection than those who received PN, less need for surgical intervention, fewer septic complications, and shorter hospital stays (mean reduction 2.9 days, 95\% CI 1.6 to 4.3 ) (table). The EN and PN groups did not differ for complications other than infections or hospital mortality (table).

\section{CONCLUSION}

Evidence from primarily low quality trials shows that in patients with acute pancreatitis, enteral nutrition reduces infections, septic

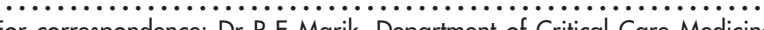
For correspondence: Dr P E Marik, Department of Critical Care Medicine, University of Pittsburgh Medical Center, Pittsburgh, PA, USA. maripe@ccm. upmc.edu

Source of funding: no external funding. complications, need for surgical intervention, and length of hospital stay compared with total parenteral nutrition but does not affect non-infectious complications or hospital mortality.

\section{Commentary}

7 he review by Marik et al recommends the use of EN for patients with AP. Based on the poor quality of the included studies, the pooled effect could be an overestimation. ${ }^{1}$ However, existing studies with various levels of evidence also provide support for the use of EN for severely ill patients. ${ }^{2}$

Patients with AP are a very specific disease population, and the issues related to providing $\mathrm{EN}$ to these patients may differ from those related to more general patient populations. For example, enteral feeding of patients with AP requires that nurses be able to recognise the link between deterioration of AP symptoms and possible dislocation of the feeding tube or symptoms related to an overload of food in the small intestine. Attention to feeding tube positioning, measurement of gastric retention and EN, and feeding pump controlled administration is important for AP patients.

Optimal EN in the intensive care unit often fails because EN gets less attention than other life saving technologies. Studies of feeding intake have shown that optimal feeding rarely is achieved in $>50 \%$ of patients. ${ }^{3}$ Therefore, the positive effects shown in efficacy studies may be diluted by the daily reality of clinical practice. The success of EN feeding will be determined largely by the quality of nursing practice. Jan M Binnekade, RN, MSc Academic Medical Centre University of Amsterdam Amsterdam, The Netherlands

1 Guyatt G, Rennie D, Hayward R, editors. Users' Guides Interactive (JAMA). Chicago, IL: JAMA Publishing Group; 2002 (accessed Sep 14, 2004). http://www.usersguides.org

2 Marik PE, Zaloga GP. Early enteral nutrition in acutely ill patients: a systematic review. Crit Care Med 2001;29:2264-70.

3 Adam S, Batson S. A study of problems associated with the delivery of enteral feed in critically ill patients in five ICUs in the UK. Intensive Care Med 1997;23:261-6.

Enteral nutrition (EN) $v$ parenteral nutrition (PN) for acute pancreatitis*

\begin{tabular}{llllll}
\hline & & \multicolumn{2}{l}{ Weighted event rates } & & \\
\cline { 3 - 4 } Outcomes at hospital discharge & Number of studies & EN & PN & RRR (95\% Cl) & NNT (CI) \\
\hline Infections & 6 & $11 \%$ & $29 \%$ & $56 \%(23$ to 75$)$ & $6(4$ to 12$)$ \\
Septic complications & 6 & $11 \%$ & $28 \%$ & $55 \%(22$ to 74$)$ & $6(4$ to 12$)$ \\
Surgical intervention & 4 & $11 \%$ & $19 \%$ & $52 \%(1$ to 77$)$ & $12(6$ to 519$)$ \\
Other non-infectious complications & 5 & $10 \%$ & $30 \%$ & $33 \%(-14$ to 60$)$ & Not significant \\
Hospital mortality & 6 & $8 \%$ & $12 \%$ & $32 \%(-38$ to 66$)$ & Not significant \\
\hline
\end{tabular}

*Abbreviations defined in glossary; RRR, NNT, and Cl calculated from data in article using a random effects model. 